\title{
Painéis de partículas com maravalha de Pinus spp. e fibra de sisal
}

\author{
Particleboard with shavings of Pinus spp. and sisal fiber
}

\author{
Matheus Roberto Cabral ${ }^{1}$, Juliano Fiorelli ${ }^{2}$, \\ Julio Cesar Machado Cravo $^{3}$ e Holmer Savastano ${ }^{4}$
}

\begin{abstract}
Resumo
Este estudo avaliou a viabilidade de uso de fibras de sisal associada à maravalha de Pinus spp. como matérias primas constituintes de painéis de partículas homogêneas. Os painéis foram produzidos com densidade de $0,500 \mathrm{~g} / \mathrm{cm}^{3}$ e $25 \%, 50 \%$ e $75 \%$ de fibra de sisal substituíram as partículas de maravalha de Pinus spp. A resina poliuretana bicomponente à base de óleo de mamona foi utilizada como aglomerante. Os painéis foram caracterizados com base nos documentos normativos NBR14810, 2006 e ASTM E1530, 2011 e suas propriedades termo-físico-mecânicas foram determinadas. Os resultados obtidos indicaram a viabilidade de produção dos painéis de partículas com maravalha de Pinus spp. e fibras de sisal e aqueles com $25 \%$ dessas fibras apresentaram propriedades termo-físico-mecânicas que atenderam as prescrições mínimas estabelecidas nos documentos normativos para painéis de baixa densidade.
\end{abstract}

Palavras-chave: Madeira, fibras vegetais, resina de mamona, painéis.

\begin{abstract}
This study evaluated the feasibility of sisal fibers associated with Pinus spp. as a constituent of homogeneous particle panels. The panels have been produced with a density of $0.500 \mathrm{~g} / \mathrm{cm}^{3}$ and $25 \%, 50 \%$ and $75 \%$ sisal fiber replaced the shavings of Pinus spp. Resin based on castor oil was used as a binder. The panels were characterized based on normative documents NBR14810, 2006 and ASTM E1530, 2011 and the thermophysical-mechanical properties were determined. The results indicated the feasibility of the production of the particle panels with the Pinus spp. and sisal fibers and those with $25 \%$ of these fibers presented thermomechanical properties that met the minimum requirements established in normative documents for low density panels.
\end{abstract}

Keywords: Wood, vegetable fibers, castor resin, panels.

\section{INTRODUÇÃO}

O progressivo consumo de madeira e a necessidade de tecnologias para a utilização de insumos considerados como biomassas, evitando a pressão sobre os ecossistemas naturais, abrem desafios para o desenvolvimento tecnológico e a inovação de novos produtos. Desta forma, a busca por materiais compósitos constituídos de subprodutos da madeira e fibras lignocelulósicas, como por exemplo, os painéis de partículas, tem despertado interesse nos meios acadêmicos e industriais (FIORELLI et al., 2014, GULER et al., 2008).

Os painéis de partículas aglomeradas são produtos compostos por materiais lignocelulósicos, usualmente madeiras ou fibras naturais que são combinados com resina sintética e então passam por processo prensagem a quente (MALONEY, 1996). Diversos estudos foram conduzidos com o objetivo de avaliar o desempenho físico-mecânico destes paneis produzidos com materiais não convencionais, como por exemplo, resíduo de kiwi (NEMLI et al., 2003), Kenaf (KALAYCIOGLU;

${ }^{1}$ Doutorando em Engenharia e Ciência de Materiais. USP - Universidade de São Paulo / Faculdade de Zootecnia e Engenharia de Alimentos. Av. Duque de Caxias Norte - 13663900 - Pirassununga, SP, Brasil, Brasil. E-mail: matheusrc@usp.br 2Professor Associado do Departamento de Engenharia de Biossistemas. USP - Universidade de São Paulo / Faculdade de Zootecnia e Engenharia de Alimentos. Av. Duque de Caxias Norte - 13663900 - Pirassununga, SP, Brasil, Brasil. E-mail: julianofiorelli@usp.br

${ }^{3}$ Doutor em Qualidade e Produtividade Animal. USP - Universidade de São Paulo / Faculdade de Zootecnia e Engenharia de Alimentos. Av. Duque de Caxias Norte - 13663900 - Pirassununga, SP, Brasil, Brasil. E-mail: juliomachadomachado@hotmail.com

${ }^{4}$ Professor Titular do Departamento de Engenharia de Biossistemas. USP - Universidade de São Paulo / Faculdade de Zootecnia e Engenharia de Alimentos. Av. Duque de Caxias Norte - 13663900 - Pirassununga, SP, Brasil, Brasil. E-mail: holmersi@usp.br 
NEMLI, 2006), cascas de amêndoa (GURU; TEKELI; BILICI, 2006), fibra de linho (PAPADOPOULOS; HAGUE, 2003), palha de trigo e miolo do milho (WANG; SUN, 2002), feixes de sisal (MESQUITA et al., 2015) e bagaço de cana-de-açúcar (FIORELLI et al., 2012).

A fibra de sisal é utilizada com frequência na produção de fios, cordas, cabos, tapetes, carpetes, colchões, tapetes e artigos artesanais. No entanto, durante as duas últimas décadas, novos usos como constituintes de materiais compósitos vêm sendo propostos para essas fibras (BISANDA; ANSELL, 1992; MOHAN; KANNY, 2012; MUKHOPADHYAY; SRIKANTA, 2008; SAHA et al., 2012).

No Brasil, a fibra de sisal é utilizada com frequência devido ao fácil cultivo e a alta produção, que no ano de 2014 foi aproximadamente 95,4 mil t (CONAB, 2014).

Visando propor novos usos para essa matéria prima, esse trabalho teve como objetivo produzir e caracterizar por meio de ensaios termo-físico-mecânicos, painéis de partículas homogêneos de maravalha de Pinus spp. e fibra de sisal aglomeradas com resina poliuretana bicomponente à base de óleo de mamona.

\section{MATERIAL E MÉTODOS}

\section{Matéria-prima}

Para fabricação dos painéis aglomerados foram adquiridas partículas de maravalha de Pinus spp. de indústrias moveleiras, localizadas nas regiões de Macatuba e São Manoel, estado de São Paulo. Os feixes de fibras de sisal (Agave Sisalana) foram adquiridos de empresas fornecedoras localizadas no estado da Bahia-Brasil.

A resina poliuretana bicomponente à base de óleo de mamona era constituída de poliol e pré-polímero.

A secagem das partículas de maravalha de Pinus spp. e das fibras de sisal foi realizada em estufa com circulação de ar forçada, a $60^{\circ} \mathrm{C}$, até atingir teor de umidade de $10 \%$. O processamento das fibras de sisal foi realizado em moinho de facas (modelo MB-20, fabricante Astecma), com peneira de até $10 \mathrm{~mm}$ de comprimento. Posteriormente, ambas as matérias primas foram classificadas separadamente em um agitador automático (modelo G, Produtest, Brasil) para obter partículas de maravalha e fibras de sisal com comprimentos de $10 \mathrm{~mm}$.

\section{Processo de produção dos painéis de partículas}

Os painéis de partículas homogêneas foram produzidos em laboratório com densidade de 0,500 $\mathrm{g} / \mathrm{cm}^{3}$ e dimensões finais de $40 \mathrm{~cm}$ x $40 \mathrm{~cm} \times 1 \mathrm{~cm}$, seguindo a metodologia de produção descrita por Maloney (1996). A proporção do adesivo utilizado foi de $12 \%$ da massa total seca das partículas. Os parâmetros de processo adotados foram $100^{\circ} \mathrm{C}$ de temperatura e $5 \mathrm{MPa}$ de pressão por 10 min. Após finalizada a prensagem, os painéis foram armazenados em temperatura ambiente por 72 h para a finalização da cura do adesivo. A Tabela 1 apresenta os tratamentos avaliados.

Tabela 1. Teor de fibra de sisal e maravalha de Pinus spp. para cada tratamento.

Table 1. Sisal fiber and Pinus spp wood content for each treatment.

\begin{tabular}{lcc}
\hline Tratamentos & Fibra de sisal (\%) & Maravalha de Pinus spp. (\%) \\
\hline T1 & 0 & 100 \\
T2 & 25 & 75 \\
T3 & 50 & 50 \\
T4 & 75 & 25 \\
T5 & 100 & 0 \\
\hline
\end{tabular}

Após a produção e cura dos painéis aglomerados de partículas foram confeccionados os corpos de prova para os ensaios de condutividade térmica e físico-mecânicos seguindo as recomendações estabelecidas pelas normativas ASTM E1530 (ASTM, 2011) e NBR 14810-3 (ABNT, 2006), respectivamente.

\section{Caracterização termo-físico-mecânicas}

Para realização do ensaio de condutividade térmica (CT) foram extraídos cinco corpos de cada um dos tratamentos em estudo (T1; T2; T3; T4 e T5). O ensaio de CT foi realizado no equipamento TA Thermal Conductivity Meter, Modelo DTC 300 seguindo a prescrição da normativa ASTM E1530 (ASTM, 2011). 
As propriedades físico-mecânicas dos tratamentos T1; T2; T3; T4 e T5 avaliadas foram: inchamento em espessura após 2 horas (IE-2h) e 24 horas (IE-24h) de imersão em água, absorção de água após 2 horas (AA-2h) e 24 horas (AA-24h) de imersão em água, módulo de elasticidade (MOE) e módulo de resistência (MOR) na flexão estática. Os ensaios foram conduzidos seguindo as recomendações da normativa brasileira ABNT (2006) (Chapas de madeira aglomerada), devido à semelhança dos painéis aglomerados avaliados nesse trabalho com os painéis de partículas de madeira.

\section{Analise microestrutural}

A microestrutura dos painéis em estudo foi realizada por meio de obtenção de imagens em microscópio eletrônico de varredura (TM-3000, Hitachi, Japão). As imagens foram geradas pela aquisição de elétrons retroespalhados com aumento de $50 \mathrm{X}$. Foram preparadas 3 amostras para cada tratamento (T1; T2; T3; T4 e T5) com dimensões de 10 x 10 x $10 \mathrm{~mm}$ aleatoriamente extraídas dos painéis em estudo. A microestrutura dos painéis foi avaliada por uma população de 75 imagens de 3 diferentes amostras.

\section{Análise estatística dos dados}

Para avaliar as propriedades termo-físico-mecânicas foram montados experimentos dispostos segundo o delineamento inteiramente ao acaso (DIC), onde o fator tratamento foi constituído em cinco níveis $(0,25,50,75$ e 100\% de fibra de sisal), totalizando em cinco tratamentos que dispunham de dez repetições.

Para todos os experimentos, o fator Tratamento foi considerado quantitativo, sendo utilizada a regressão quando a ANOVA foi significativa. Os coeficientes das equações foram testados pelo Teste $T$, enquanto que o coeficiente de determinação $\left(R^{2}\right)$ dos experimentos foi obtido através da razão entre a soma de quadrados de Regressão e soma de quadrados de Tratamentos (NOGUEIRA, 2007).

Para executar a ANOVA dos dados experimentais foi realizada a verificação da homogeneidade e normalidade dos erros experimentais (NOGUEIRA, 2007). Após essa verificação foi aplicado o teste de Shapiro Wilk (normalidade) e de Bartlett (homogeneidade de variâncias) para as propriedades investigadas. O software utilizado para interpretar os resultados foi o programa R estatística versão 2.5.1.

\section{RESULTADOS E DISCUSSÃO}

\section{Análise descritiva das propriedades termo-físico-mecânicas dos painéis}

A Tabela 2 apresenta os valores médios e desvio padrão do inchamento em espessura (IE) $2 \mathrm{~h}$ e 24 h, absorção de água (AA) 2h e 24h, módulo de ruptura (MOR), módulo de elasticidade (MOE) e condutividade térmica (CT). Visualiza-se que os valores médios para as propriedades físicas e mecânicas variaram entre os tratamentos, enquanto que para a condutividade térmica não foi constatado variação. Com relação à dispersão dos dados, observa-se que as propriedades físicas e mecânicas exibiram alta variação. Provavelmente, esse resultado pode ter relação com a baixa dispersão da resina entre as partículas e com a variação da geométrica das partículas de maravalha de Pinus spp. e fibras de sisal, uma vez que apresentam diferentes razões de aspecto.

Tabela 2. Propriedades termo-físico-mecânicas médias de painéis de partículas homogêneas de maravalha de Pinus spp. e fibra de sisal.

Table 2. Average thermal-physical-mechanical properties of homogeneous particleboard panels of Pinus spp. and sisal fiber.

\begin{tabular}{lccccccc}
\hline Tratamento & $\begin{array}{c}\text { AA 2h } \\
\mathbf{( \% )}\end{array}$ & $\begin{array}{c}\text { AA 24h } \\
\mathbf{( \% )}\end{array}$ & $\begin{array}{c}\text { IE 2h } \\
\mathbf{( \% )}\end{array}$ & $\begin{array}{c}\text { IE 24h } \\
\mathbf{( \% )}\end{array}$ & $\begin{array}{c}\text { MOR } \\
\text { (MPa) }\end{array}$ & $\begin{array}{c}\text { MOE } \\
\text { (MPa) }\end{array}$ & $\begin{array}{c}\text { CT } \\
\text { (W/mK) }\end{array}$ \\
\hline \multirow{2}{*}{$\mathrm{T} 1$} & 90,70 & 100,45 & 11,18 & 11,77 & 8,73 & 1266,72 & 0,12 \\
& $\pm 15,39$ & $\pm 46,77$ & $\pm 2,51$ & $\pm 2,43$ & $\pm 2,00$ & $\pm 264,32$ & $\pm 0,008$ \\
\hline \multirow{2}{*}{ T2 } & 62,36 & 96,17 & 12,05 & 19,39 & 9,49 & 1246,27 & 0,11 \\
& $\pm 11,18$ & $\pm 15,72$ & $\pm 5,40$ & $\pm 8,70$ & $\pm 2,08$ & $\pm 133,47$ & $\pm 0,010$ \\
\hline \multirow{2}{*}{ T3 } & 73,84 & 98,33 & 17,78 & 19,80 & 8,02 & 1143,83 & 0,11 \\
& $\pm 15,53$ & $\pm 16,24$ & $\pm 6,39$ & $\pm 5,34$ & $\pm 3,97$ & $\pm 413,47$ & $\pm 0,005$ \\
\hline \multirow{2}{*}{ T4 } & 93,31 & 126,55 & 20.39 & 26.46 & 6,71 & 1053.07 & 0,11 \\
& $\pm 31,60$ & $\pm 24,29$ & $\pm 6,08$ & $\pm 5,21$ & $\pm 2,72$ & $\pm 349,46$ & $\pm 0,005$ \\
\hline \multirow{2}{*}{ T5 } & 100.13 & 128,88 & 23,56 & 25.83 & 5,54 & 1249.35 & 0,11 \\
& $\pm 29,30$ & $\pm 29,60$ & $\pm 7,14$ & $\pm 10,47$ & $\pm 1,81$ & $\pm 212,64$ & $\pm 0,005$ \\
\hline
\end{tabular}




\section{Análise inferencial das propriedades termo-físico-mecânicas}

A Tabela 3 descreve os P-valores dos testes de normalidade (Shapiro Wilk) e homogeneidade (Bartlett) das propriedades investigadas. Observa-se que os erros das variáveis experimentais exibiram P-valores acima de $\mathrm{p}<0,05$ para ambos os testes, o que caracteriza os resíduos como normais e homogêneos. No entanto, para a variável AA 2h, foi necessário adotar a transformação de dados, uma vez que, segundo Nogueira (2007), quando os dados originais não atenderem as pressuposições para ANOVA, funções são construídas para que as pressuposições sejam atendidas por essas funções. Portanto, para essa variável foi utilizada a transformação $\sqrt{y}$.

Tabela 3. Análise de Pressuposição ANOVA.

Table 3. Assumptions Analysis ANOVA.

\begin{tabular}{ccc}
\hline Variáveis & Normalidade & Homogeneidade \\
\hline AA 2h & $0,288^{\mathrm{NS}}$ & $0,067^{\mathrm{NS}}$ \\
AA 24h & $0,599^{\mathrm{NS}}$ & $0,124^{\mathrm{NS}}$ \\
IE 2h & $0,184^{\mathrm{NS}}$ & $0,109^{\mathrm{NS}}$ \\
IE 24h & $0,106^{\mathrm{NS}}$ & $0,215^{\mathrm{NS}}$ \\
MOR & $0,664^{\mathrm{NS}}$ & $0,204^{\mathrm{NS}}$ \\
MOE & $0,370^{\mathrm{NS}}$ & $0,064^{\mathrm{NS}}$ \\
CT & $0,154^{\mathrm{NS}}$ & $0,078^{\mathrm{NS}}$ \\
\hline
\end{tabular}

NS - Não significativo a $P<0,05$

\section{Propriedades físicas}

Para as propriedades físicas, a ANOVA revelou significância $(\mathrm{P}<0,05)$ para todas as variáveis estudadas, demonstrando que a inclusão de fibra sisal influencia as propriedades físicas de painéis de partículas homogêneas de maravalha de Pinus spp.

Na Figura 1, as variáveis AA 24h, IE $2 \mathrm{~h}$ e IE $24 \mathrm{~h}$ apresentaram efeito linear crescente $(\mathrm{P}<0,05)$, enquanto que a variável AA $2 \mathrm{~h}$ exibiu efeito cúbico $(\mathrm{P}<0,05)$. Nota-se, que o menor $\mathrm{R}^{2}$ foi verificado para AA 24h, enquanto que as demais variáveis físicas exibiram altos valores. Os coeficientes da equação da AA $2 \mathrm{~h}$ (Figura 1a) foram utilizados como base nos valores transformados em $\sqrt{y}$. No entanto, os valores médios experimentais apresentados foram transformados para os valores originais.
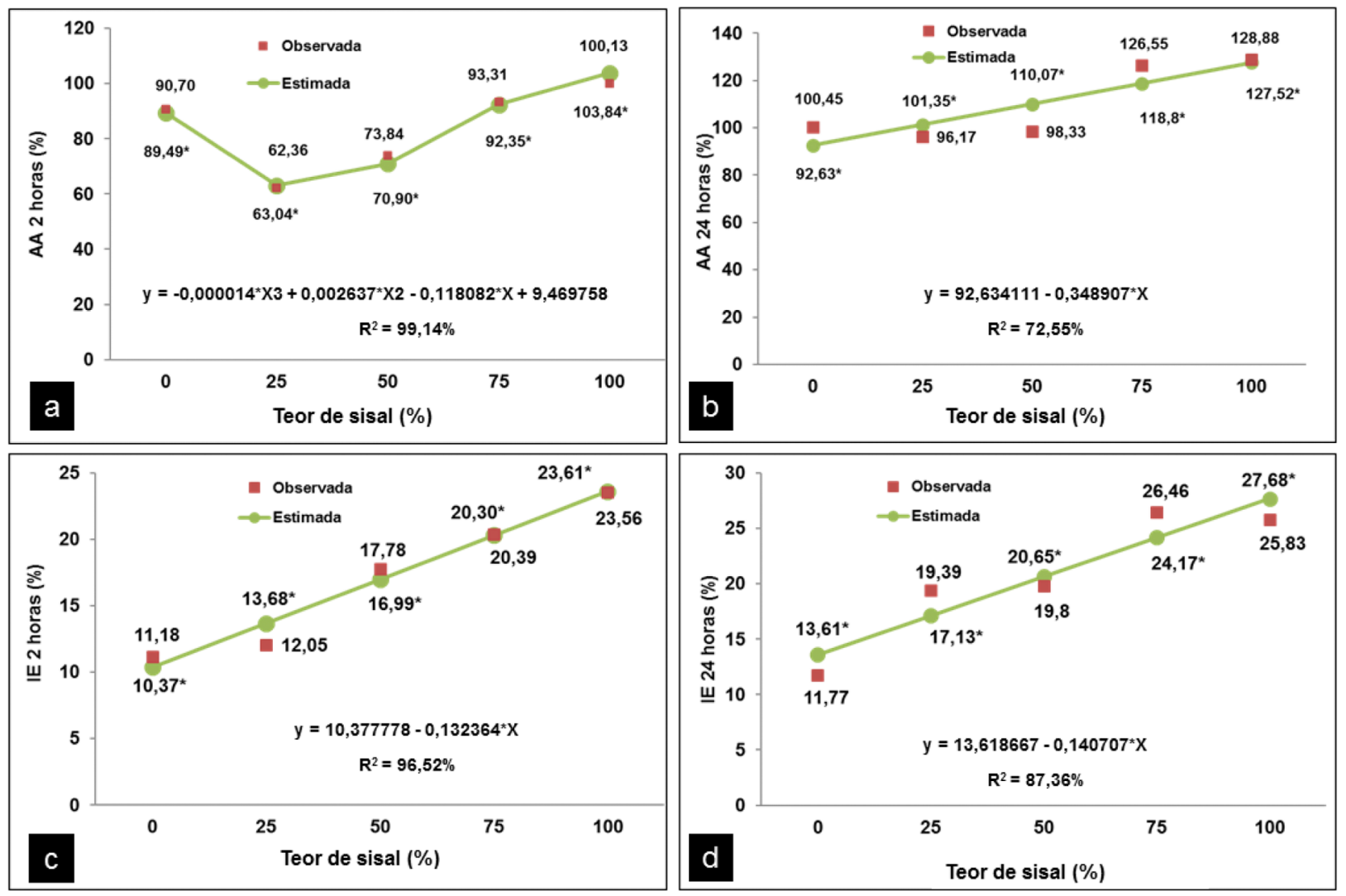

Figura 1. Variação das propriedades físicas com diferentes teores de fibras de sisal: a) AA 2 h; b) AA 24 h; c) IE 2 $h$; d) 24 h.

Figure 1. Variation of physical properties with different sisal fibers contents: a) AA 2 h; b) AA 24 h; c) IE 2 h; d) 24 h. 
A AA $24 \mathrm{~h}$, IE $2 \mathrm{~h}$ e IE $24 \mathrm{~h}$ dos painéis foram significativamente menores nos painéis que continham em sua formulação somente maravalha (T1), enquanto que os maiores valores foram constatados para os painéis com fibra de sisal (T5). Quando a fibra de sisal é incorporada aos painéis de maravalha (T2, T3 e T4), essas variáveis físicas aumentam significativamente $(\mathrm{P}<0,05)$. Esse fenômeno pode estar atrelado à diferentes razões de aspecto (Tabela 4 e figura 2) das partículas de maravalha de Pinus spp. e fibra de sisal. Essa característica das partículas pode ter influenciado na distribuição do adesivo no momento do processo de produção e, consequentemente, nas propriedades físicas dos painéis (MALONEY, 1996), contribuindo com o aumento do volume de vazios e da porosidade.

Tabela 4. Razão de aspecto da maravalha de Pinus spp. e das fibras de sisal.

Table 4. Pinus spp. and sisal fibers aspect ratio.

\begin{tabular}{lccc}
\hline & $\mathbf{L}(\mathbf{m m})$ & $\mathbf{d}(\mathbf{m m})$ & $\mathbf{L} / \mathbf{d}$ \\
\hline Maravalha Pinus spp & 8,0 & 5,0 & 1,5 \\
Fibras de Sisal & 8,0 & 0,15 & 53,3 \\
\hline
\end{tabular}
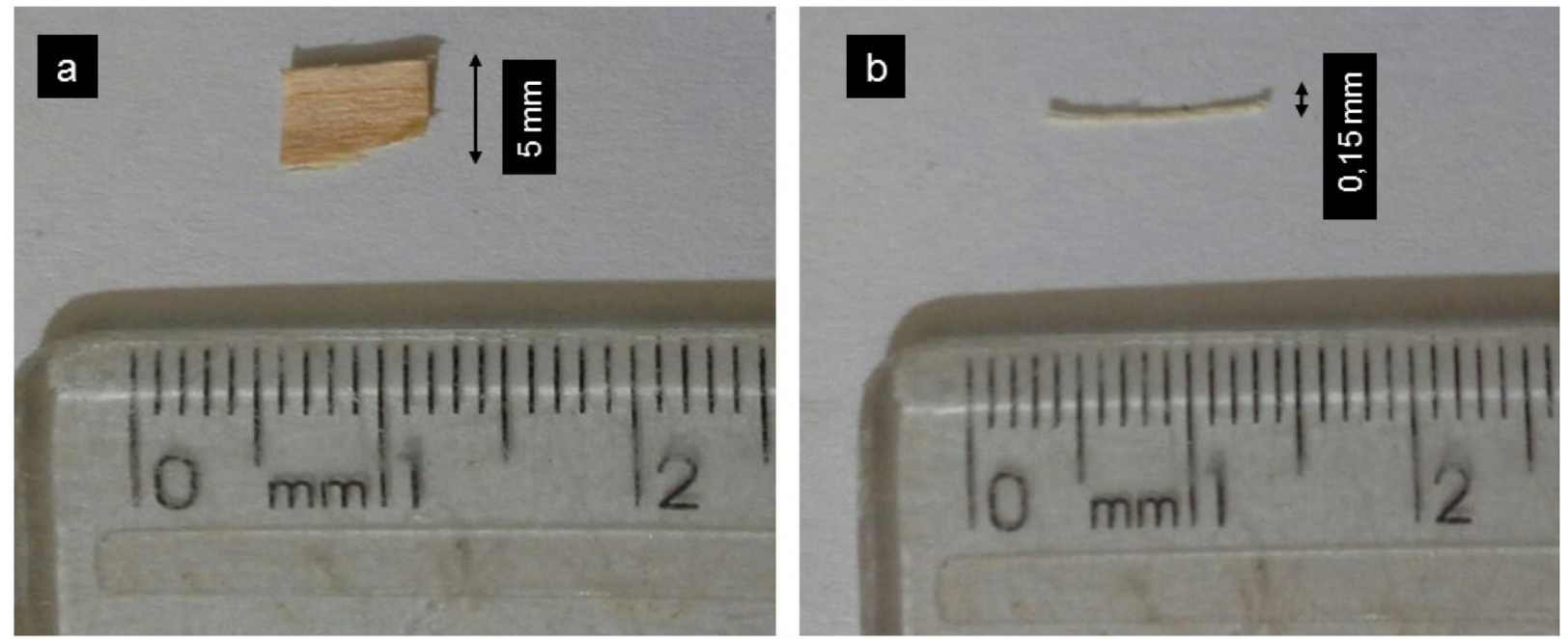

Figura 2. a) maravalha Pinus spp; b) fibra de sisal

Figure 2. a) shaving of Pinus spp; b) sisal fiber

Além disso, outra hipótese que pode ser adicionada na discussão desses resultados físicos tem relação com o caráter hidrofílico das fibras de sisal (BARRA et al., 2015). A hidrofílicidade do sisal pode ter acarretado nos resultados superiores para AA $2 \mathrm{~h}$ e $24 \mathrm{~h}$, bem como IE $2 \mathrm{~h}$ e $24 \mathrm{~h}$ dos painéis com maiores teores dessa fibra, visto que essa matéria prima tem potencial de absorção de água, devido ao elevado teor de microfibrilas de celulose (aproximadamente $78 \%$ ), e do baixo teor de lignina (aproximadamente 7\%) (BARRETO et al., 2011; BISMARCK et al., 2002).

Resultados experimentais desse estudo apresentam semelhanças com os obtidos para painéis de partículas de madeira (Eucalyptus grandis) com adição de casca de arroz (Oryza Sativa L.) na proporção de 0, 20, 40, 60, 80 e 100\% (MELO et al., 2009). Os pesquisadores constataram que o IE no período de 2 e $24 \mathrm{~h}$ foi crescente em função do aumento da taxa de casca de arroz nas partículas de madeira. Scatolino et al. (2013) avaliaram a incorporação de sabugo de milho $(0,25,50$, 75 e 100\%) em painéis de Pinus oocarpa e foi observado que os valores de AA 24h aumentaram de $74 \%$ para 95\%, à medida que havia acréscimo de sabugo de milho. Mendes et al. (2010) avaliaram a influência da incorporação de casca de café $(25,50$ e 75\%) em painéis de partículas de madeira (Eucalyptus urophylla) e foi constatado o incremento dos resultados físicos de AA 24h e IE 24h.

Comportamento discrepante ocorreu para AA $2 \mathrm{~h}$ (Figura 1 a) que apresentou redução significativa $(\mathrm{P}<0,05)$ para os tratamentos com 25\% (T2) e 50\% (T3) de fibras de sisal, em comparação com o tratamento (T1). A explicação para esse resultado pode ter relação com o procedimento de seleção das amostras, as quais podem apresentar um maior acumulo de resina nos espaços vazios, fator relacionado com a deficiência da dispersão da resina durante o processo de produção devido as diferentes razões de aspecto das partículas. Apesar da fibra de sisal ter afetado as propriedades 
físicas dos painéis, os valores de IE $2 \mathrm{~h}$ e IE $24 \mathrm{~h}$ apresentaram valores abaixo dos $35 \%$ preconizados pelo documento normativo ANSI A208.1 (ANSI, 1999). Esses resultados demonstram que a fibra de sisal apresenta potencial para utilização como matéria-prima para fabricação de painéis particulados em associação com maravalha de Pinus spp.

\section{Propriedades Mecânicas}

Com relação às propriedades mecânicas, a ANOVA exibiu efeito significativo $(P<0,05)$ somente para o MOR. Assim, a adição de fibras de sisal em painéis de partículas de maravalha de Pinus spp. interferiu nessa propriedade mecânica. A Figura 2 ilustra que a incorporação de fibras de sisal reduz significativamente $(\mathrm{P}<0,05)$ o MOR, uma vez que foi registrado efeito linear decrescente. Os resultados desse estudo demostraram que os painéis produzidos com 100\% de fibras de sisal (T5) apresentaram valor médio de MOR 36,54\% inferior quando comparado com os painéis fabricados com $100 \%$ de partículas de maravalha (T1).

Para as propriedades mecânicas de painéis de partículas aglomeradas, um fator importante a ser considerado, é a composição química da matéria prima. Segundo Melo Filho et al. (2013) o conteúdo de lignina presente nas fibras de sisal é de aproximadamente 7\%, teor este relativamente baixo quando comparado com o teor de lignina presente em maravalha de Pinus spp. de aproximadamente $29 \%$ (FIORELLI et al., 2014).

De acordo com estudos apresentados por Rios et al. (2015) e Joseleau et al. (2004) o elevado teor de lignina pode contribuir significativamente com a adesão entre as partículas, uma vez que em temperaturas elevadas $\left(100^{\circ} \mathrm{C}\right)$ a lignina se liquidifica e atua como um plastificante, favorecendo a adesão das partículas e a distribuição das tensões no compósito, refletindo em melhores propriedades mecânicas.

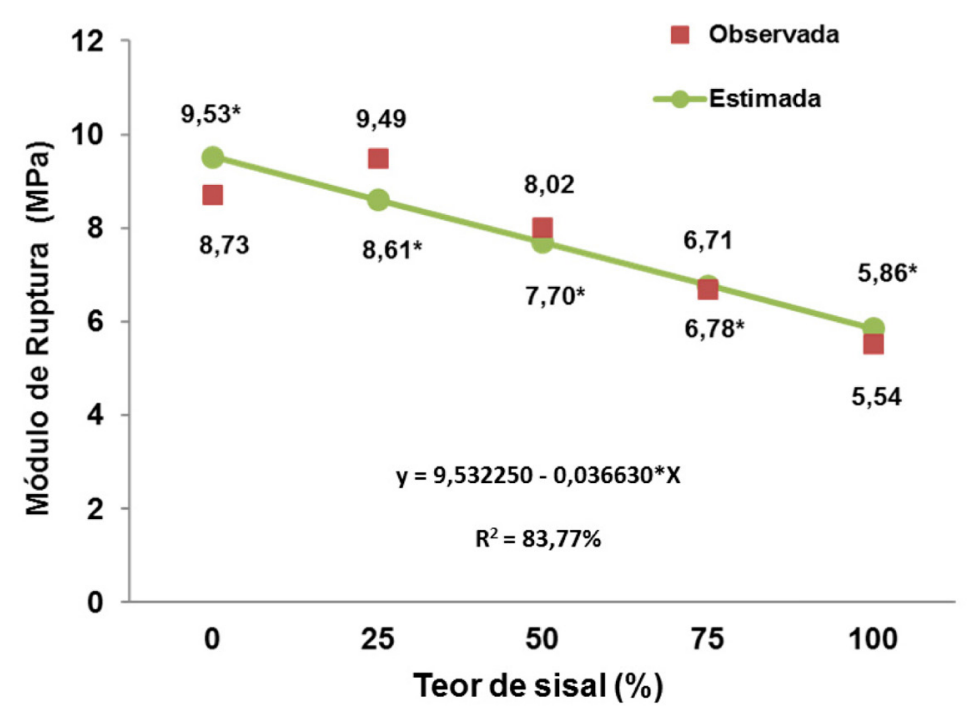

Figura 3. Variação do MOR para tratamentos com diferentes teores de sisal. Figure 3. Variation of MOR for treatments with different contents of sisal.

As propriedades mecânicas médias dos tratamentos foram comparadas (Figura 4) com os valores estabelecidos na normativa ANSI A208 (ANSI, 1999) para painéis de partículas de baixa densidade $\left(0,64 \mathrm{~g} / \mathrm{cm}^{3}\right)$. Segundo esse documento normativo, os valores mínimos de MOR e MOE são 3,0 $\mathrm{MPa}$ e $550 \mathrm{MPa}$, respectivamente. Os painéis experimentais (T1, T2, T3, T4 e T5) apresentaram valores superiores ao preconizado pelo documento normativo. Embora a inclusão de fibra de sisal em substituição a maravalha de Pinus spp. tenha afetado negativamente o MOR dos painéis em estudo, todos os tratamentos avaliados possuem potencial para produção. 


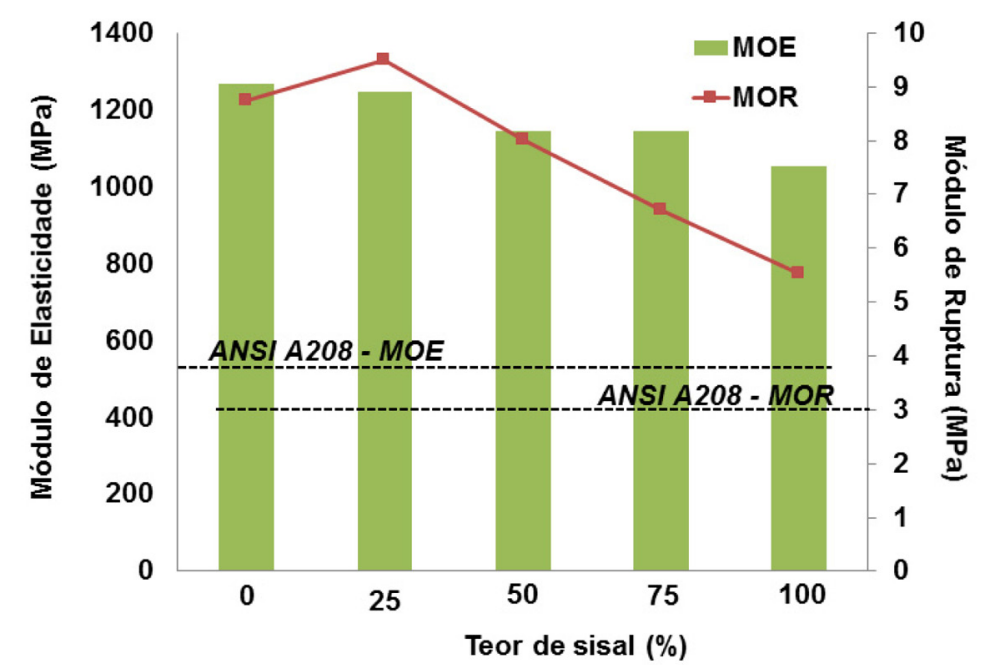

Figura 4. Propriedades mecânicas experimentais e normativa para painéis de baixa densidade. Figure 4. Experimental and normative mechanical properties for low density panels.

\section{Propriedade térmica}

$\mathrm{Na}$ análise da ANOVA não foi constatado efeito significativo $(\mathrm{P}<0,05)$ para a variável condutividade térmica, revelando que os tratamentos em estudo não apresentaram diferença estatística $(\mathrm{P}<0,05)$. Segundo Wang (1988) materiais que apresentem condutividade térmica menor que 0,25 $\mathrm{W} / \mathrm{mK}$ são considerados isolantes térmicos. Pela Figura 5 todos os tratamentos apresentaram valores abaixo de $0,25 \mathrm{~W} / \mathrm{mk}$ e portanto são classificados como material isolante.

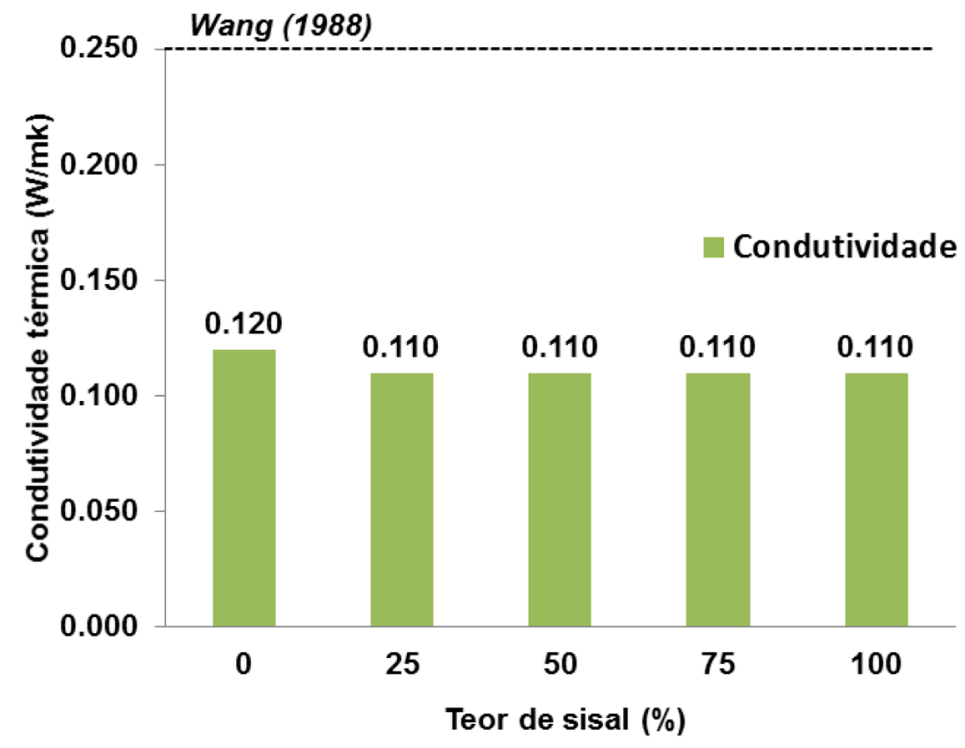

Figura 5. Valores médios de condutividade térmica experimental comparado com literatura. Figure 5. Mean values of experimental thermal conductivity compared to literature.

\section{Caracterização microestrutural}

Na Figura 6 estão apresentadas as imagens representativas da microestrutura dos painéis experimentais. Nessas imagens, é possível identificar a distribuição das partículas de maravalha, fibra de sisal e poros. A variação da geometria das partículas que apresentam diferentes razões de aspecto (tabela 2) contribuiu com o aumento da porosidade dos painéis constituídos de maravalha de Pinus spp. e fibra de sisal.

Segundo Melo e Menezzi (2010) a absorção de água nos painéis de partículas ocorre através dos espaços vazios presentes no interior do material. Sendo assim, as imagens de MEV possibilitam explicar o aumento significativo da absorção de água e do inchamento em espessura dos tratamentos em estudo constituídos de fibra de sisal. 

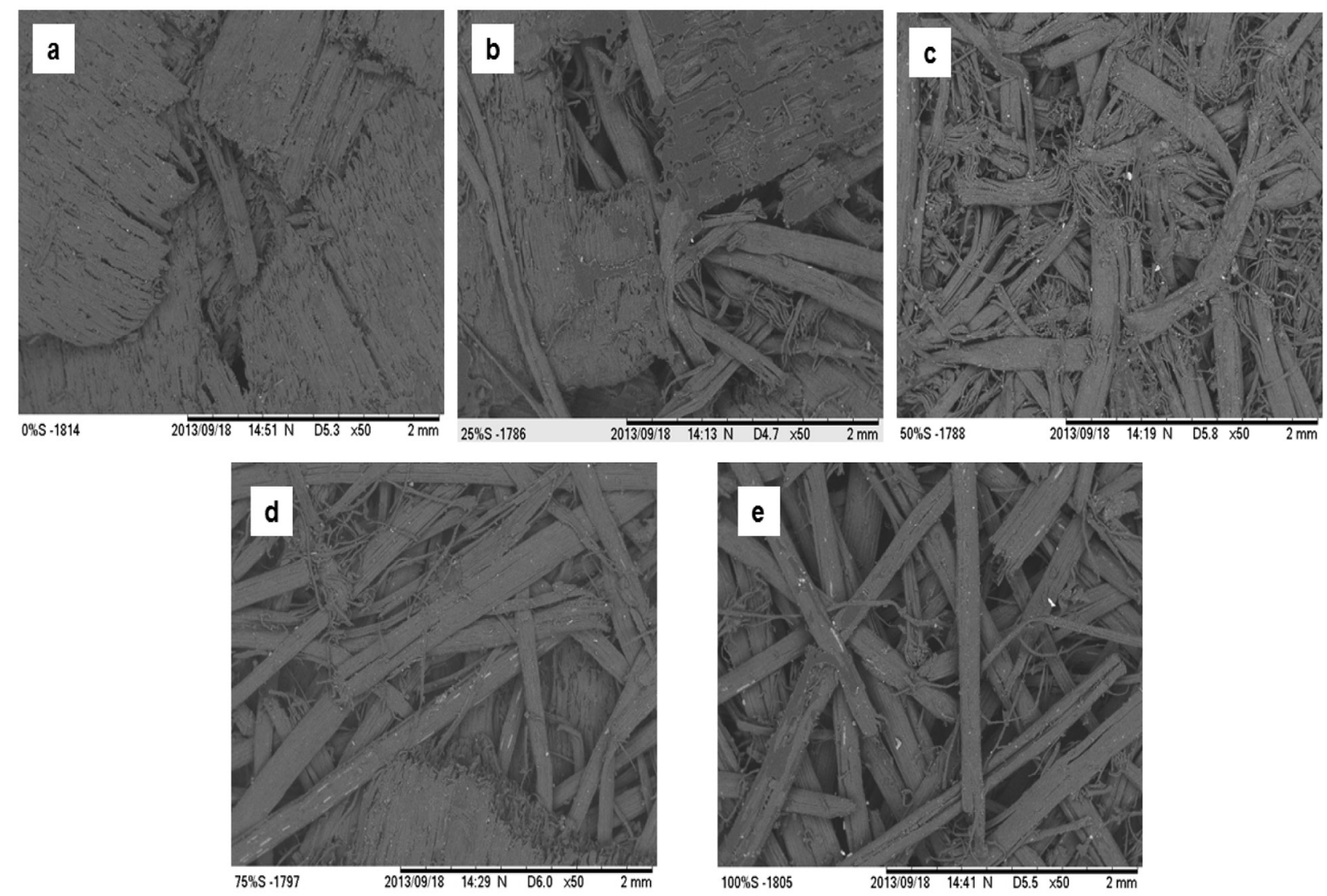

Figura 6. Imagens de MEV de painéis de partículas com maravalha de Pinus spp. e fibra de sisal ampliadas 50x. a) T1, b) T2, c) T3 d) T4, e) T5.

Figure 6. SEM Images of particleboard with shavings of Pinus spp. sisal fiber increased 50x. a) T1, b) T2, c) T3 d) T4, e) T5.

\section{CONCLUSÕES}

Esse estudo avaliou o potencial de uso de fibra de sisal como matéria-prima para fabricação de painéis particulados associada à maravalha de Pinus spp. e as seguintes conclusões foram obtidas:

- Os painéis com fibra de sisal apresentaram valores médios de absorção de água e inchamento em espessura superiores aos aferidos para painéis com 100\% de maravalha de Pinus spp. que tem relação com a variação na razão de aspecto das partículas e na hidrofilicidade da fibra de sisal;

- Os tratamentos avaliados atenderam as recomendações mínimas de propriedades físico-mecânicas para painéis de baixa densidade estabelecidas pelos documentos normativos internacionais;

- Os painéis avaliados são classificados como materiais isolantes;

- Os painéis constituídos de 75\% de maravalha de Pinus spp. e 25\% de fibra de sisal apresentaram propriedades físico-mecânicas que o qualifica como a melhor formulação avaliada.

\section{AGRADECIMENTOS}

Os autores agradecem o apoio financeiro concedido pela FAPESP (processo 2012/13881-2, processo 2012/18635-0 e processo 2012/51467-3), CNPq, ao programa de pós-graduação em Engenharia e Ciência de Materiais da Universidade São Paulo e ao Núcleo de Pesquisa em Materiais para Biossistemas-BioSMat.

\section{REFERÊNCIAS BIBLIOGRÁFICAS}

ABNT - ASSOCIAÇÃO BRASILEIRA DE NORMA TÉCNICAS. NBR 14810-3: Chapas de madeira aglomerada, Parte 3: Métodos de Ensaio, terminologia. Rio de Janeiro, 2006. 
ASTM - AMERICAN SOCIETY FOR TESTING AND MATERIALS. ASTM E1530-11 - Evaluating the resistance to thermal transmission of materials by the guarded heat flow meter technique. West Conshohocken, 2011.

ANSI - AMERICAN NATIONAL STANDARDS INSTITUTE. A208.1 - Standard. American National Standard Particleboard. Washington, 1999.

BARRA, B. N.; SANTOS, S. F.; BERGO, P.; V A. ALVES, C.; GHAVAMI, K.; SAVASTANO, H. Residual sisal fibers treated by methane cold plasma discharge for potential application in cement based material. Industrial Crops and Products, v. 77, p. 691-702, 2015.

BARRETO, A. C H.; ROSA, D. S.; FECHINE, P. B A.; MAZZETTO, S. E. Properties of sisal fibers treated by alkali solution and their application into cardanol-based biocomposites. Composites Part A: Applied Science and Manufacturing, v. 42, n. 5, p. 492-500, 2011.

BISANDA, E. T. N.; ANSELL, M. P. Properties of sisal-CNSL composites. Journal of Materials Science, Dordrecht, v. 27, n. 6, p. 1690-1700, 1992.

BISMARCK, ALEXANDER.; ARANBEFWI-ASKARGORTA, IBON.; SPRINGER, JÜRGEN.; LAMPKE, THOMAS.; WIELAGE, BERNHARD.; STAMBOULIS, ARTEMIS.; SHENDEROVICH, ILJA.; LIMBACH, HANS-HEINRICH. Surface Characterization of Flax, Hemp and Cellulose Fibers; Surface Properties and the Water Uptake Behavior. Polymer Composites, v. 23, n. 5, p. 872-894, 2002.

CONAB - COMPANHIA NACIONAL DE ABASTECIMENTO. Sisal 2014 Produção, Exportações e Preços em alta. Brasília: CONAB, 2014.

FIORELLI, J.; GOMIDE, ABDALA, CATARINA.; LAHR, F. A. R.; NASCIMENTO, M. F.; SARTORI, D.; BALLESTEROS, J. E. MEJIA.; BUENO, BONILA, STEPHEN.; BELINI, U. L. Physico-chemical and anatomical characterization of residual lignocellulosic fibers. Cellulose, v. 21, n. 5, p. 3269-3277, 2014.

FIORELLI, J.; DONIZETTI, D.; BARRERO, N. G.; SAVASTANO, H.; AGNOLON, E.; JOHNSON, R. Particulate composite based on coconut fiber and castor oil polyurethane adhesive: An eco-efficient product. Industrial Crops and Products, v. 40, p. 69-75, 2012.

GULER, C., COPUR, Y., TASCIOGLU, C. The manufacture of particleboards using mixture of peanut hull (Arachis hypoqaea L.) and European Black pine (Pinus nigra Arnold) wood chips. Bioresource Technology, Essex, v. 99, n. 8, p. 2893-2897, 2008.

GURU, M.; TEKELI, S.; BILICI, I. Manufacturing of urea-formaldehyde-based composite particleboard from almond shell. Materials and Design, v. 27, n. 10, p. 1148-1151, 2006.

JOSELEAU, J. P.; IMAI, T.; K. K.; RUEL, K. Detection in situ and characterization of lignin in the G-layer of tension wood fibres of Populus deltoides. Planta, Berlin, v. 219, n. 2, p. 338-345, 2004.

KALAYCIOGLU, H.; NEMLI, G. Producing composite particleboard from kenaf (Hibiscus cannabinus L.) stalks. Industrial Crops and Products, v. 24, n. 2, p. 177-180, 2006.

MALONEY, T. M. The family of wood composite materials. Forest Products Journal. Madison, v. 46, n. 2, p. 19-26. 1996.

MELO, R. R.; DEL MENEZZI, C. Influência da Massa Específica nas Propriedades Físico-Mecânicas de Painéis Aglomerados Influence of the Density in Physical-Mechanical Properties of Particleboards. Silva Lusitana, Lisboa, v. 18, n. 1, p. 59-73, 2010. 
Cabral et al. - Painéis de partículas com maravalha de Pinus spp. e fibra de sisal

MELO, R. R.; SANTINI, E. J.; HASELEIN, C. R.; STANGERLIN, D. M. Propriedades físico-mecânicas de painéis aglomerados produzidos com diferentes proporções de madeira e casca de arroz. Ciência Florestal, Santa Maria, v 19, n 4, p. 449-460, 2009.

MELO FILHO, J. A.; SILVA, F. DE A.; TOLEDO FILHO, R. D. Degradation kinetics and aging mechanisms on sisal fiber cement composite systems. Cement and Concrete Composites, v. 40, p. 30-39, 2013.

MENDES, R. F.; MENDES, L. M.; BENEDITO, J. J, G.; MORI, F.A.; CESAR, A, A. Effect of the incorporation of coffee husks on the physico-mechanical properties of Eucalyptus urophylla ST Blake particleboards. Ciência e Agrotecnologia, Lavras, v. 34, n. 2008, p. 610-617, 2010.

MESQUITA, R. G. A; MENDES, L. M.; MENDES, R.F. TONOLI, G.D.; MARCONCINI, J.M. Inclusão de feixes de sisal na produção de painéis MDP de eucalipto Inclusion of sisal bundles in the production of eucalyptus MDP panels. Scientia Forestalis, v. 43, n. 105 p.75-82, 2015.

MOHAN, T. P.; KANNY, K. Chemical treatment of sisal fiber using alkali and clay method. Composites Part A: Applied Science and Manufacturing, v. 43, n. 11, p. 1989-1998, 2012.

MUKHOPADHYAY, S.; SRIKANTA, R. Effect of ageing of sisal fibres on properties of sisal - Polypropylene composites. Polymer Degradation and Stability, v. 93, n. 11, p. 2048-2051, 2008.

NEMLI, G.; HÜSEYIN K.; SERDAR, B.; NURGÜL A. Suitability of kiwi (Actinidia sinensis Planch.) prunings for particleboard manufacturing. Industrial Crops and Products, v. 17, n. 1, p. 39-46, 2003.

NOGUEIRA, M. C. S. Experimentação Agronômica I. Piracicaba: Universidade de São Paulo, 2007.

PAPADOPOULOS, A. N.; HAGUE, J. R. B. The potential for using flax (Linum usitatissimum L.) shiv as a lignocellulosic raw material for particleboard. Industrial Crops and Products, v. 17, n. 2, p. 143-147, 2003.

RIOS, P. D. A.; VIEIRA, H. C.; STUPP, Â. M. Avaliação física e mecânica de painéis reconstituídos compostos por partículas de galhos secos de Araucaria angustifolia (Bertol) Kuntze e madeira de Eucalyptus grandis Hill ex Maiden Physical and mechanical review of particleboard. Scientia Forestalis, Piracicaba, v. 43, n. 106, p. 283-289, 2015.

SAHA, P.; MANNA, S.; SEN, R.; ROY, D.; ADHIKARI, B. Durability of lignocellulosic fibers treated with vegetable oil-phenolic resin. Carbohydrate Polymers, v. 87, n. 2, p. 1628-1636, 2012.

SCATOLINO, M. V.; WISKY, D. S.; MENDES, R. F.; MENDES, L.M. Use of maize cob for production Ciência e Agrotecnologia, Lavras, v. 37, n. 4, p. 330-337, 2013.

WANG, S. H. Construction Materials Science. Beijing: China Construction Industry Publisher, 1988.

WANG, D.; SUN, X. S. Low density particleboard from wheat straw and corn pith. Industrial Crops and Products, v. 15, n. 1, p. 43-50, 2002.

Recebido em 06/03/2016

Aceito para publicação em 06/03/2017 